\title{
Could a Sentinel Lymph Node Biopsy Be Performed with Isolated Methylene Blue Injection in a Patient with a History of Skin-Sparing Mastectomy and Prosthetic Reconstruction?
}

\author{
Ümit Özdemir ${ }^{1}$ (D) İhsan Burak Karakaya ${ }^{1}$ (D) Ahmet Karayiğit ${ }^{2}$ (D) \\ Dursun Burak Özdemir² (D) Hayrettin Dizen² ${ }^{2}$ \\ 1 Eskişehir Osmangazi University Medical Faculty, Department of General Surgery, Section of Gastroenterological Surgery, Eskişehir, Turkey. \\ 2 Eskişehir Osmangazi University Medical Faculty, Department of General Surgery, Section of Surgical Oncology, Eskişehir, Turkey.
}

\begin{abstract}
Evaluation of lymphatic spread in early stage breast cancer without clinical and radiological evidence of metastasis can be performed by sentinel lymph node biopsy (SLNB). However, controversies about performing the SLNB in patients who have a history of major breast or axillary region surgery keep going. This case report presents the outcomes of a SLNB performed on a 42-year-old woman who had been previously treated with bilateral nipple and skin-sparing mastectomy and breast reconstruction with areolar complex transfer and silicone breast implants. $0.5 \%$ diluted methylene blue solution was injected intradermally as a marker. SLNB is an inexpensive and effective method for adequate axillary evaluation in cases with previous mastectomy history. Intradermal injection of $0.5 \%$ diluted methylene blue could reduce the risk of skin necrosis and breast prosthesis rupture.
\end{abstract}

Key words: Sentinel Lymph Node Biopsy, Breast Cancer, Methylene Blue, Previous Skin-Sparing Mastectomy.

\section{INTRODUCTION}

Sentinel lymph node biopsy (SLNB) is the standard assessment method of the lymphatic system used in earlystage (T1 or T2) breast cancer patients when axillary lymph nodes are clinically negative (1). If there is no evidence of malignancy in the SLNB of patients undergoing surgery due to breast cancer, then axillary lymph node dissection (ALND), which causes high morbidity, may not be performed (2). Methylene blue, isosulfan blue and radioisotope materials could be used in SLNB for marking lymphatic tissue (3). After an excisional biopsy of breast tumors, it is reported that SLNB is an appropriate technique for determining the lymphoid metastases (4). However, performing SLNB in patients who have previously undergone major breast or axilla surgery is still controversial $(5,6)$. 
This case report aims to present the results of performing SLNB by using intradermal methylene blue on a 42-yearold woman who had been previously treated with bilateral nipple and skin-sparing mastectomy and breast reconstruction.

\section{CASE REPORT}

A 42-year-old woman in the premenopausal period who had no family history of breast cancer revealed lesions that were radiologically typed as BIRADS 3 in the left breast during malignancy screening. It was noticed that the patient gave birth twice and continued to breastfeed after both pregnancies for two years. After an evaluation performed at the plastic surgery clinic for breast masses, a bilateral skin and areola sparing mastectomy was performed. Breast reconstruction was added to the procedure by using prepectoral implants. In the pathological evaluation, a solid lesion was detected at the upper left part of the left breast, and the largest diameter of the tumor was $9 \mathrm{~mm}$. Surgical margins at the anterior, posterior, and lateral sides were 1 , 5 , and $10 \mathrm{~mm}$, respectively. As a result of the histological evaluation, the patient was diagnosed with invasive ductal carcinoma.

No axillary involvement was detected by ultrasonography and PET/CT evaluations. The patient was referred to a general surgery outpatient clinic after evaluation of the pathological examination results. The multidisciplinary oncology council concluded that the clinical and radiological evaluation of the axillary lymph node area of the patient was not sufficient due to the pathological diagnosis. Axillary lymph node dissection may cause many morbidities, especially lymphedema and nerve injuries in the arm. In order to avoid the morbidity of ALND, SLNB was planned as the first procedure to evaluate the lymphatic system for the extent of ductal carcinoma invasion.

Since the entire breast parenchyma had been excised, the methylene blue solution was diluted due to the fact that a $1 \%$ solution could cause necrosis on the skin. The $0.5 \%$ methylene blue solution was injected equally into each periareolar quadrant intradermally. In order to facilitate the transition of the injected solution to the lymphatic system, gentle breast massage was performed from the areola towards the axilla for five minutes.

The axillary region was exposed by using an incision parallel to the lateral margin of the pectoralis major muscle in order to reach the sentinel lymph node region. Blue stained lymphatics were found and the blue lymph nodes were excised after the clavipectoral fascia was dissected (Figure 1). Histopathological evaluation revealed that none of the sentinel lymph nodes were positive for malignancy involvement (Figure 2). There was no hyperemia, ischemia, or necrosis on the skin during the postoperative follow-up (Figures 3 and 4). The patient was referred to the radiation oncology clinic for entire breast and axilla radiotherapy.

Figure 1: axillery incision after the blue lymph nodes were excised

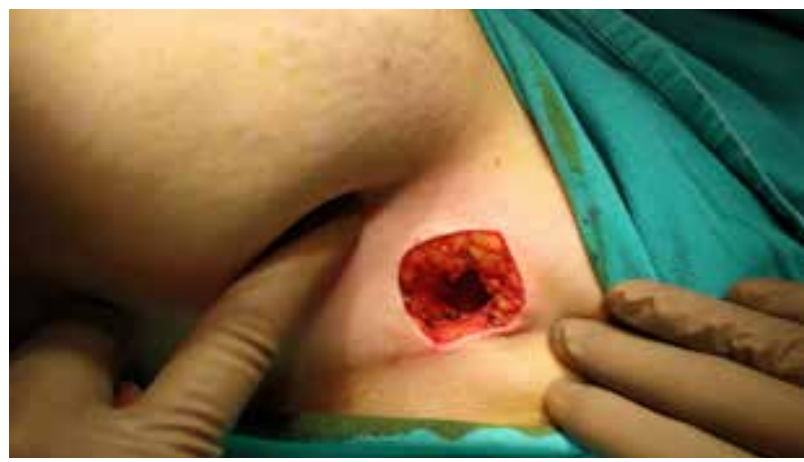

Figure 2: the surgical specimen

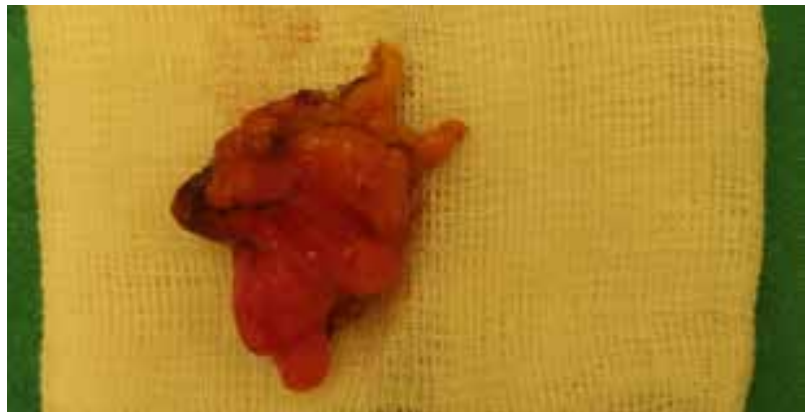

Figure 3: There was no hyperemia, ischemia or necrosis after the operation.

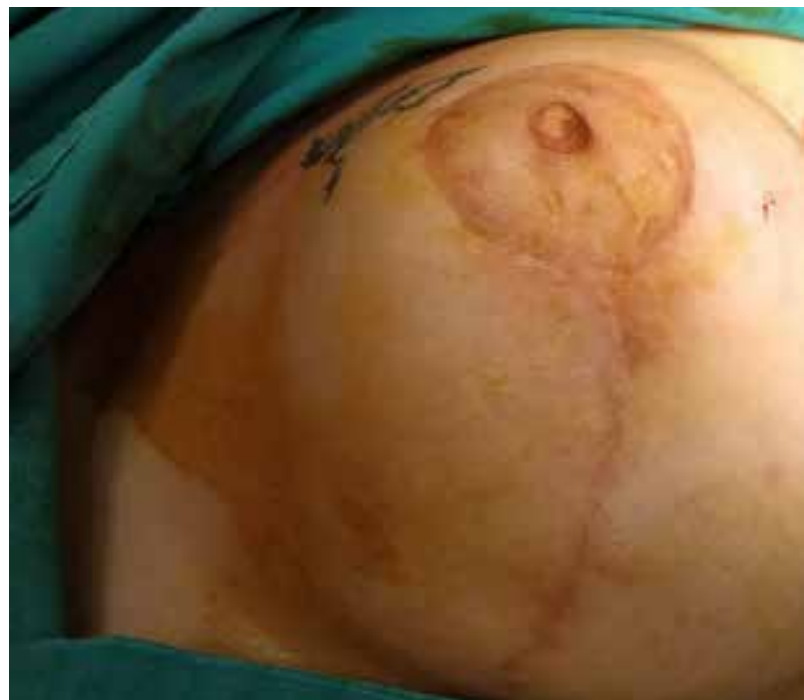


Figure 4: There was no hyperemia, ischemia or necrosis after 2 mounts.

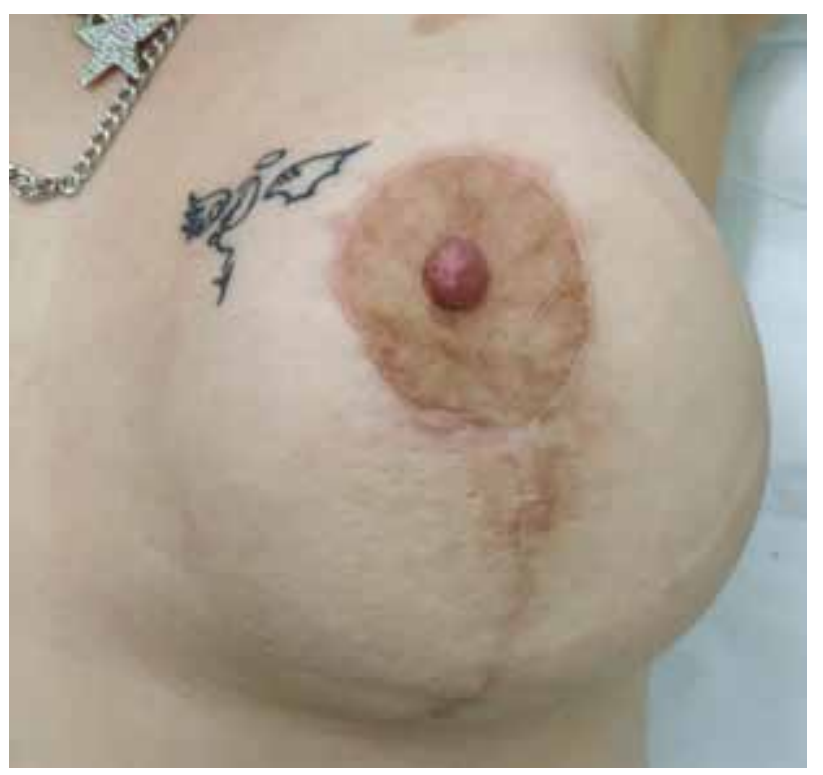

\section{DISCUSSION}

SLNB is based on the theory that lymphatic flow in the breast goes directly to a single lymph node in the axilla. Therefore, it is controversial to perform SLNB in patients who have previously undergone breast or axilla surgery. The reliability of SLNB after an incisional biopsy in the breast was investigated by Çelebioğlu et al., and recognition of the sentinel lymph nodes and false negativity rates are reported similar to primary excisions (4). In patients who have previously undergone breast and axilla surgery, the recognition ratio of the sentinel lymph node in SLNB is approximately $60 \%$, and there is still benefit in performing it. However, because the lymphatic drainage pattern is likely to change, doing it together with lymphoscintigraphy could decrease the false negativity rate $(5,7)$. Despite this, a lymphoscintigraphy and gamma probe could not be performed on the current patient due to changing hospital conditions during the COVID-19 pandemic. In addition, the value of subsequent SLNB is not clear in patients undergoing cosmetic breast surgery (reduction mammoplasty, breast augmentation) (8-10).

In the current case, axillary surgery was not performed, but the breast parenchyma was completely excised. In a study by Karam et al., 20 patients who had previously undergone mastectomy underwent SLNB due to recurrence. Sentinel lymph nodes were identified in 13 of them. In the aforementioned study, a radioisotope was used in each patient as a marker, and blue dye was added in some of the patients (11). In another study by Intra et al, SLNB was performed on four patients who had previously undergone mastectomy without axillary surgery due to ductal carcinoma in situ (DCIS). Sentinel lymph nodes were identified in all of four patients, and ALND was performed on two of them due to positive results from the SLNB. In this study, radioisotopes were used as markers, and blue dyes were not used (12). In the case presented by Vicente et al, SLNB was performed using radioisotopes and isosulfan blue as markers on a patient who underwent mastectomy five years previously due to DCIS. The operation was completed with ALND, since all three lymph nodes that were removed were positive (13).

In the literature, radioisotope substances have frequently been used as markers in SLNB after mastectomy. It has also been reported in the literature that skin necrosis may develop due to intradermal administration of methylene blue (14).

In the current case, sentinel lymph nodes were diagnosed with only intradermal methylene blue injection without using any radioisotopes. During the procedure or followup period, there were no complications related to the skin or breast implant because methylene blue was administered intradermally at a lower dilution (0.5\%).

SLNB with intradermal injection of $0.5 \%$ isolated methylene blue solution is an easy, practical, inexpensive, and effective alternative technique that could be performed on patients with a history of mastectomy in specialized centers on oncological surgery.

\section{Declarations}

The authors received no financial support for the research and/or authorship of this article. There is no conflict of interest. 


\section{REFERENCES}

1. Lyman GH, Somerfield MR, Bosserman LD, Perkins CL, Weaver DL, Giuliano AE. Sentinel Lymph Node Biopsy for Patients With EarlyStage Breast Cancer: American Society of Clinical Oncology Clinical Practice Guideline Update. Journal of clinical oncology : official journal of the American Society of Clinical Oncology. 2017;35(5):561-4.

2. Rao R, Euhus D, Mayo HG, Balch C. Axillary node interventions in breast cancer: a systematic review. Jama. 2013;310(13):1385-94.

3. McMasters KM, Tuttle TM, Carlson DJ, Brown CM, Noyes RD, Glaser $\mathrm{RL}$, et al. Sentinel lymph node biopsy for breast cancer: a suitable alternative to routine axillary dissection in multi-institutional practice when optimal technique is used. Journal of Clinical Oncology. 2000;18(13):2560-6.

4. Celebioglu F, Frisell J, Danielsson R, Bergkvist L. Sentinel node biopsy in non-palpable breast cancer and in patients with a previous diagnostic excision. European journal of surgical oncology : the journal of the European Society of Surgical Oncology and the British Association of Surgical Oncology. 2007;33(3):276-80.

5. Ahmed M, Baker R, Rubio IT. Meta-analysis of aberrant lymphatic drainage in recurrent breast cancer. The British journal of surgery. 2016;103(12):1579-88

6. Poodt IGM, Vugts G, Schipper RJ, Roumen RMH, Rutten HJT, Maaskant-Braat AJG, et al. Prognostic impact of repeat sentinel lymph node biopsy in patients with ipsilateral breast tumour recurrence. The British journal of surgery. 2019;106(5):574-85.

7. Karanlik H, Ozgur I, Kilic B, Fathalizadeh A, Sanli Y, Onder S, et al. Sentinel lymph node biopsy and aberrant lymphatic drainage in recurrent breast cancer: Findings likely to change treatment decisions. Journal of surgical oncology. 2016;114(7):796-802.

8. Munhoz AM, Aldrighi C, Buschpiegel C, Ono C, Montag E, Fells K, et al. The feasibility of sentinel lymph node detection in patients with previous transaxillary implant breast augmentation: preliminary results. Aesthetic plastic surgery. 2005;29(3):163-8.

9. Gray RJ, Forstner-Barthell AW, Pockaj BA, Schild SE, Halyard MY. Breast-conserving therapy and sentinel lymph node biopsy are feasible in cancer patients with previous implant breast augmentation. The American journal of surgery. 2004;188(2):122-5.

10. Mottura AA, Del Castillo R. Transaxillary Breast Augmentation: Two Breast Cancer Patients with Successful Sentinel Lymph Node Diagnosis. Aesthetic Plastic Surgery. 2007;31(5):544-9.

11. Karam A, Stempel M, Cody III HS, Port ER. Reoperative sentinel lymph node biopsy after previous mastectomy. Journal of the American College of Surgeons. 2008;207(4):543-8.

12. Intra M, Veronesi $P$, Gentilini OD, Trifirò G, Berrettini A, Cecilio R, et al. Sentinel lymph node biopsy is feasible even after total mastectomy. Journal of surgical oncology. 2007;95(2):175-9.

13. Vicente DA, Henry LR, Hahm G, Soballe PW, Smart D. Axillary sentinel lymph node biopsy after mastectomy: a case report. World Journal of Surgical Oncology. 2010;8(1):59.

14. Bleicher RJ, Kloth DD, Robinson D, Axelrod P. Inflammatory cutaneous adverse effects of methylene blue dye injection for lymphatic mapping/sentinel lymphadenectomy. Journal of surgical oncology. 2009;99(6):356-60. 